\title{
INTERACTIONAL OBJECTS: HCI CONCERNS IN THE ANALYSIS PHASE OF THE SYMPHONY METHOD
}

\author{
Guillaume Godet-Bar, Dominique Rieu, Sophie Dupuy-Chessa, David Juras \\ LIG Laboratory - 681 rue de la Passerelle \\ BP 72 - 38402 St Martin d'Hères - France \\ FirstName.LastName@imag.fr
}

\begin{abstract}
Keywords: $\quad$ Augmented Reality, Design method, Interactional Objects, HCI, Software Engineering.
Abstract: We present in this paper a set of concepts that extend a design method issued from the Software Engineering domain, in order to take into account Human-Computer Interaction design, in particular for Augmented Reality systems. Previous works focused on the initial phases of development (i.e., Specification phases). Our efforts concentrate on the Analysis phase, into which we have introduced a new concept - Interactional Objects- that allows designers to structure the interactional space, and a specific relation that permits to draw links between the business and interactional spaces. These contributions also enable developers to develop reusable components and encourage code generation.
\end{abstract}

\section{INTRODUCTION}

The evolution of computer technologies, in terms of communication (wireless networking) and interaction device (visualization headsets, tactile gloves) deeply alter the classical, implicit perception of Human-Computer Interaction (HCI). The user can now evolve in environments blending real and virtual entities. We shall use the concept of "Augmented Reality system" to designate any interactive system that superimposes virtual data onto the real world. These systems must address major challenges for their development and use, such as the cohabitation of physical and numerical spaces, multiple and complex interactions between these worlds (variety of device, multimodality, usability). Neither HCI's design methods and evaluation practices, nor Software Engineering's (SE) tools and techniques are adapted to these specific contexts.

Our goal is to propose a design method integrating both SE methods for the development of the functional core and HCI practices for designing the interaction. The design of the system's functionalities should therefore rely on well-known models such as UML, which is supported by several design processes, for instance the Rationale Unified
Process (Jacobson et al., 1999). The proposed method must also allow the development of classical interactive systems as well as integrate specific activities necessary to the development of Augmented Reality systems. We address this problem by extending an existing design method: Symphony, into which we introduce new design phases, new concepts - Interactional Objects - and new models that provide a bridge and an adaptation between SE and HCI concepts. This paper focuses in particular on the latter.

In the next section, we present the Symphony method, used as a medium for merging $\mathrm{HCI}$ and $\mathrm{SE}$ approaches, and a case study from which several examples are extracted throughout the paper. The third section details our contributions as an addendum to the process previously introduced. In the fourth section, we address the problem of design for reuse and how it applies in the context of our contribution.

\section{RELATED WORK}

\subsection{Models for HCI}

Our design method is based on models for SE and for HCI. For SE, we use the UML standard. In 
HCI, design is often based on task analysis. So classical models in HCI are task trees such as ConcurrTask Trees (Paternó, 2003).

For augmented reality systems, models such as ASUR (Dubois et al., 2002), IRVO (Chalon and David, 2004) have been proposed to take into account their interactional specificities. These models aim to complement classical approaches. For example, ASUR or IRVO present possible interactions in the context of a user task described using a task model. User tasks correspond to the abstract or actual actions a system user may perform, such as select an object, move it around the graphical interface...

\subsection{Design methods}

Being based on different models and processes, compatibility between design methods for interactive systems and for the functional core is a recurring problem that has already been subject to specific studies (Tarby 2001), (Lim 1994). In particular, (Gulliksen and Göransson, 2005) and (Sousa and Furtado, 2003) propose to extend the Rationale Unified Process with the design of interaction, in a user-centred approach. (Constantine et al., 2003) also describe a process unifying the design of interaction and that of the functional core but in a usage-centred approach. None of these works addresses Augmented Reality-specific aspects, such as the representation of interaction device like Head-Mounted Displays, positioning systems... Moreover, they offer a weak formalization of proposed processes, which makes them difficult to apply for developers.

\section{SYMPHONY}

\subsection{General concepts}

In this section we introduce an extension of the Symphony design method, used as a medium for merging HCI and SE development processes.

Symphony is a user-oriented, business component-based development process originally proposed by the UMANIS company. It has already been extended by (Hassine et al., 2002) and (Juras et al., 2006a), mainly in order to improve reusability of components, and lately to integrate the design of complex interfaces such as those featuring Augmented Reality systems.
Symphony is organized into three design branches, similarly to 2TUP, into a Y-lifecycle. The whole lifecycle is applied for each functional unit of the system under development (see below):

- The functional (left) branch corresponds to the traditional task of domain and user requirements modelling, independently from technical aspects,

- The technical (right) branch allows developers to design both the technical and applicative architectures. It also federates all the constraints and technical choices with relation to security, pervasiveness, load balancing...

- The central branch integrates the technical and functional branches into the design model, which merges the analysis model with the applicative architecture and details traceable components.

Organization of phases in Symphony is summarized in Figure 1. For the sake of conciseness, we will only cover in this section a few aspects of a system's design (functional branch) through the Specification and Analysis phases of the extended Symphony process.

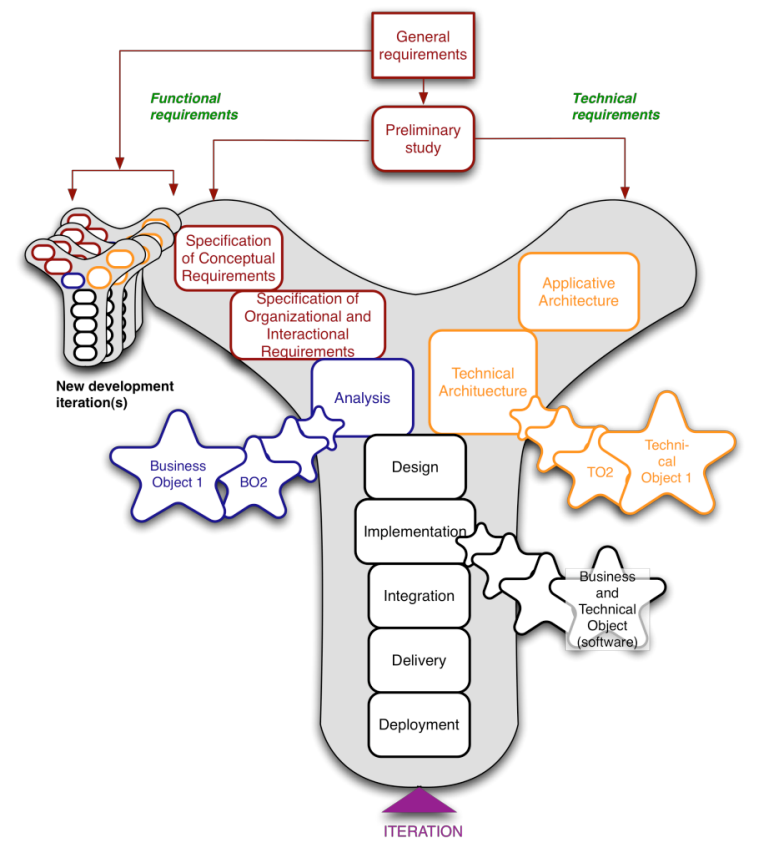

Figure 1: Symphony design phases

All phases aim at refining models and scenarios previously outlined. SE and HCI-oriented activities are realized in parallel, by design actors specialized either in Software Engineering or Human-Computer Interaction. However, both may collaborate in order to ensure consistency of adopted design options, as detailed in (Juras et al., 2006b). One will note that mappings between models exist but are not described in this paper. 


\subsection{Case study}

We chose to address a well-known problematic encountered by real estate agents when making an inventory of fixtures: the scarcity of data available to evaluate a housing. Indeed, most of real estate business processes feature basic computerization: specifically, details about damages are in most cases lay out as paper forms and textual descriptions.

Such data is often insufficient when the real estate agent needs to evaluate the evolution of a specific damage or wearing out from one occupation to the next, especially when this is a contentious issue between the tenants and the expert or the landholder.

One solution would consist in allowing the agent to visualize directly the past and current states of the premise, using visual cues to signal elements that need special attention. An Augmented Reality interface comes to mind when it comes to designing such a system.

In the following sections, we detail the development process we adopted for this application.

\subsection{Specification of Conceptual Requirements}

As a prologue to the Specification of Conceptual Requirements phase, the Preliminary study essentially deals with splitting up the system requirements into independent functional units: Business Processes (i.e., a Business Process can be considered as a collection of activities taken as a response to a specific type of input or event and produces an output of value for the process' client). Each is assigned a whole iteration of the Y-lifecycle and development priorities. Actors (e.g., landholder, expert, tenants) interacting with the Business Process are also identified.

During the Specification of Conceptual Requirements phase, Business Processes are described in terms of nominal scenario and highlevel sequence diagrams where only actors and Business Process are represented (i.e., there s no decomposition of the system yet). We shall focus in this paper on the "Management of inventories of fixtures" Business Process.

Business Processes are then refined so as to identify uninterrupted exchanges between actors and the Business Process. Such units constitute Business Processes Components(BPC), which are themselves described using scenario and sequence diagrams. These descriptions include alternative scenarios (extensions of the nominal scenario).

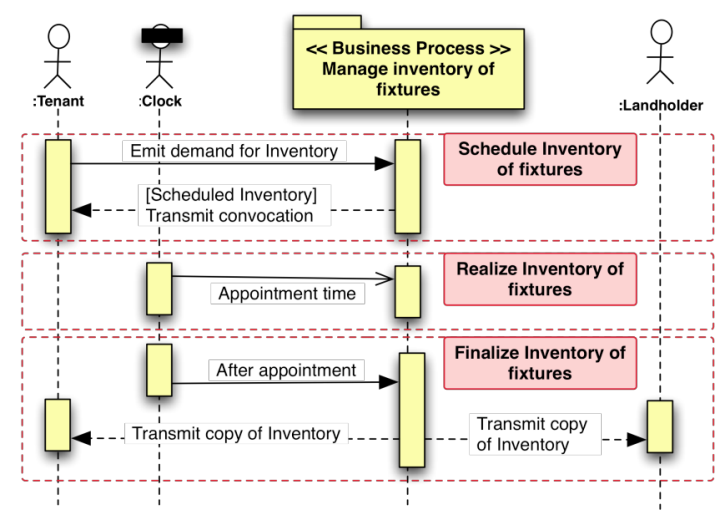

Figure 2: Sequence diagram for the Manage Inventory of fixtures Business Process

Successive refinements of the "Management of the inventories of fixtures" Business Process have led to identifying the "Realize an inventory of fixtures" Business Process Component (Figure 2).

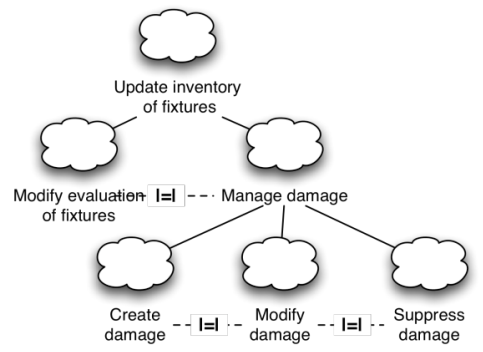

Figure 3: Extract from the task tree for the inventory of fixtures application

Parallel to the construction of sequence diagrams during the Specification of Conceptual Requirements phase, task trees (Paternó, 2003) describe abstract user tasks, for each Business Process and BPC, that is without mentioning actual device used or modalities such as text, speech, pointing... Figure 3 features an extract from the general task tree that focuses on the tasks involving the manipulation of the "Damage" concept. Tasks are ordered into a hierarchy of abstraction, with the lowest task representing refinement (and successive reification steps) of parent tasks. The " $=\mid$ " operators linking tasks at a same abstraction level indicate alternatives for the user. One will note that the task tree presented in Figure 3 does not yet feature details on how the damages are supposed to be manipulated. 


\subsection{Specification of Organizational and Interactional Requirements}

\subsubsection{Organizational Requirements}

During this activity, each Business Process Component is further refined into activity diagrams showing internal actors (for example, the Expert) and their interactions with the system and the external actors (for example, the tenants). This allows identifying manual and computerized tasks. The latter are then generalized into Use Cases.

Finally, all identified Use Cases are organized into logical packages usually representing the Business Process Component (Figure 4).

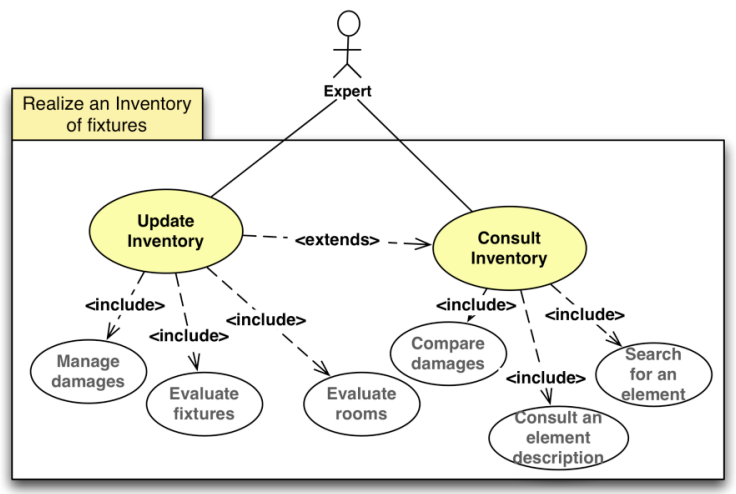

Figure 4: Organization of Use Cases into packages

\subsubsection{Interactional Requirements}

The Interactional Requirements refines abstract user task trees into concrete tasks, thus detailing device and interaction languages (e.g., vocal commands, gesture input...) manipulated by the users. Usability concerns are also addressed during this phase.

Additional models help describe and design the envisaged interaction, depending on the complexity of the future system's interface. Our case study featuring an Augmented Reality interface, we resort to ASUR models (Dubois et al., 2002) to assist the design of interaction. ASUR essentially allows the designer to describe device, mechanical relations between device, real objects used to interact with the system, users and numerical objects used as virtual representations of real entities. Relations traced between these concepts finalize the representation of the system's Human-Computer Interaction. Figure 5 thus details parts of the interaction technique used for the task "Manage Damage" in Figure 3.

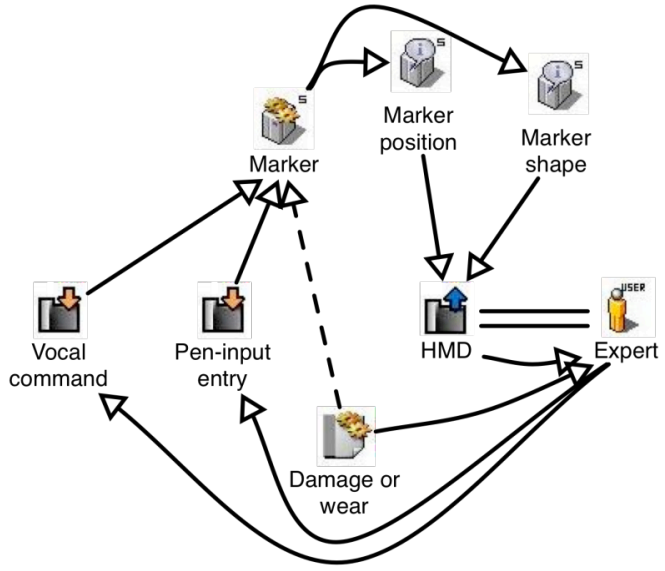

Figure 5: ASUR model as a representation of the task "Manage Damage"

In the figure above the user, identified as the Expert, is wearing a Head-Mounted Display ("==" relation) which provides information ( $\rightarrow$ relation symbolizes physical or numerical data transfer) about the position and shape of a Marker virtual object. The Marker is a numerical representation ("->" relation) of a physical Damage in the physical world. The Expert can interact with the Marker object using Vocal command and Pen-input entries.

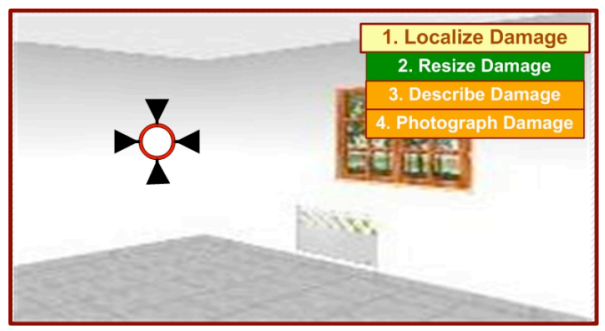

Figure 6: Interface prototype as projected on the Head-Mounted Display

Figure 6 represents an interface prototype deduced from both the task tree described in Figure 3 and the ASUR diagram illustrated in Figure 5, as should be displayed on the Head-Mounted Display. The Marker object indeed helps the user identifying damages (possible actions are described in the topright menu) while walking around the premise.

\subsection{Analysis}

This phase is quite activity-dense in the Symphony method. Two types of studies are carried out: structural (static) and dynamic analysis. The latter 
consists in refining the Use Cases into detailed scenarios and sequence diagrams (similarly to the process described in the Rational Unified Process), in order to identify logic entities: Business Objects.

The dynamic analysis only allows outlining the services (i.e., the interface) that these entities are supposed to provide, for example: adding a Marker object, defining its properties...

During the structural analysis, the Business Objects identified are expounded in terms of Interface, Master, Part and Role classes and then organized. We describe in the following paragraphs such activities.

Symphony conceptualizes the system as an assembly of independent and interconnected Business Objects. This guarantees a modularity of specifications and encourages their reuse.

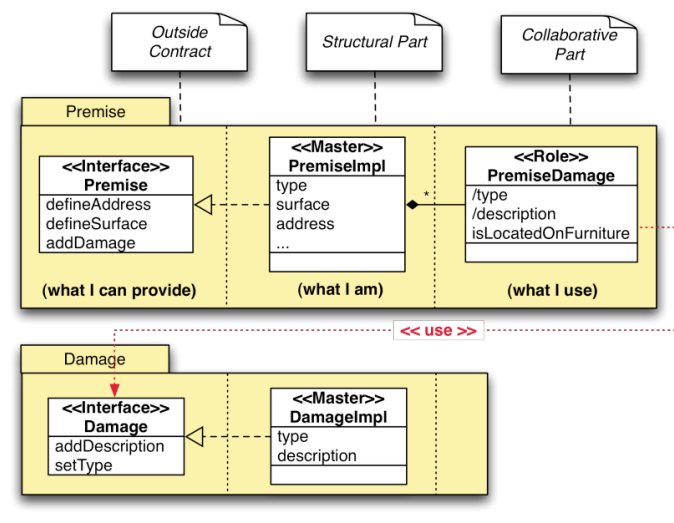

Figure 7: Example of organization between two Business Object

Figure 7 presents an example of Business Object as described during the Analysis phase. One will note its representation as a tripartite UML package, into which classical UML stereotyped classes are defined. The three parts of the Business Object are conceptually analogous to that of a UML component ${ }^{1}$. They are composed of:

An outside contract, into which an Interface class describes the services that can be provided by the object. For example, the Premise Business Object can be required to define its address, its surface or add a damage description,

A structural part, composed of two types of classes: the Master class, which is a central, autonomous part, which keeps all the knowledge about the object's manipulations, and implements the Interface ; if needed a Part class (in Figure 8,

UML superstructure $\quad-\quad$ version $2.0 \quad$ http://www.omg.org/cgi-bin/doc?formal/05-07-04 the "Localization" class) may complement the Master class in order to structure its attributes and highlight key concepts of the Master class.

A collaborative part, where Role classes feature service providers for the client object (i.e., another Business Object) and their adaptation to the object's requirements. In Figure 7, the "PremiseDamage" role is in fact an adaptation of a generic "Damage" Business Object. The slashprefixed attribute "type" describes a concept derived from the "Damage" object, while the "isLocatedOnFurniture" attribute represents a notion proper to the "PremiseDamage" role.

Amongst the different relation types which enable the designer to organize Business Objects, the "use" relation, allows developers to represent the adaptation between a given Business Object and its role as another's collaborator (i.e., an object able to provide services defined into the "Role" class). Figure 7 illustrates such an organization between Business Objects, in which the "PremiseDamage" role's adaptation of the "Damage" Business Object is clearly represented.

The interactional aspects of the Analysis phase are treated in current versions of the Symphony process as an unspecified technical layer, usually using applicative architectures such as Struts. We address the problematic of a more structured approach to interaction design in the following paragraphs.

\section{INTERACTIONAL OBJECTS AND CONCEPT MAPPING}

\subsection{Interactional concepts}

The decisions taken during the specification phases contribute to constructing an interactional space, as opposed to the traditional business space. Indeed, we saw in the previous section that the elaboration of the human-computer interaction generates new concepts that need to be integrated into the system (e.g., the Marker object as a representation of a Damage). These concepts should facilitate co-design between SE and HCI developers, as well as reuse and generation efforts already explored for the business space. Additionally, they emerge from interactional diagrams such as ASUR or more implicit notions that do not appear in these models. In that respect, we identify three cases: 
Explicit mapping: Some concepts, which are represented in the interaction diagrams previously elaborated (e.g., see Figure 5), are mappings of business concerns into the interactional space: for example, a "Marker" is a mapping of the "Damage" notion. One will note that these mappings translate properties from Business Objects into wholly different modalities: for example, the type of damage (wearing out or alteration) is represented by different marker shapes and/or colours.

Implicit mapping: Other concepts emerge from implicit aspects of the interaction diagrams. For example, in order to show the "Marker" objects to the user, it is necessary to maintain a numerical representation of the premise (a 3D mesh, or even a simple 2D plan) where the markers will be positioned. However, the numerical representation also corresponds to the "Premise" concept introduced in the business space.

No mapping: Finally, other aspects of the interactional space are neither represented in ASUR diagrams nor in the business space. For example, it is necessary to identify the user's position and orientation into the premise (in fact, of the numerical representation of the premise, see above) using passive sensors (GPS positioning, gyroscopes, triangulation... that were not represented in the ASUR diagram, essentially because they are common to most Augmented Reality systems). Defining the user's position in the numerical world is necessary so as to display the correct virtual point of view on the HMD's screens. However, this concept of "Avatar" has no equivalent into the business space, as there is no to identify the application's current user (i.e., the Expert), at least when considering the "Realize an Inventory of Fixtures" Business Process.

From a practical point of view, the mappings between the interactional and business spaces would be more clearly and easily expressed if the same models structures both spaces. Therefore, a set of evolutions needs to be undertaken concerning the Analysis phase, in order to take into account the models described during the Specification of Interactional Requirements phase, on one hand. On the other hand, it is also necessary to combine $\mathrm{HCI}$ models with the technical, Software Engineeringoriented aspects usually described during the Analysis phase, in order to provide a common ground for describing HCI and SE concepts. The following paragraphs describe such addenda.

\subsection{Interactional Objects}

In analogy with Business Objects, which constitute a conceptual view of the system's functional aspects, within a business space, we introduce the concept of Interactional Objects, which correspond to a more technical or programmatic view of the system's interactional aspects, within an interactional space. They are technology-independent models that should not put excessive constraints on the development practices of HCI designers.

As a comparison, one will note that other notations aim at structuring the interactional space. For instance, as an extension of the ASUR model for Augmented Reality, (Dubois et al., 2006) proposes a participatory design process and ASUR-IL: a transformation of ASUR components into MVCbased software units, which also allows for rapid prototyping of the system. However, this approach does not provide any formalization of the design process or mapping with SE concepts. Also, apart from restraining development to MVC-based architectures, reuse is not addressed in this work.

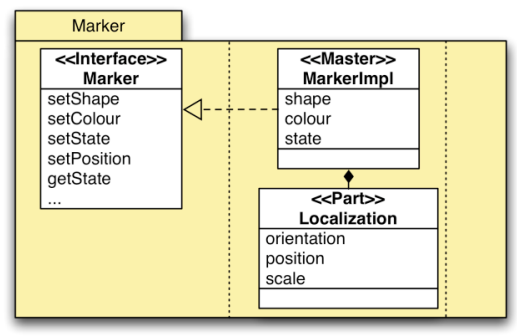

Figure 8: Interactional Object example

Despite the distinct conceptual spaces to whom they are attached, Interactional Objects and Business Objects are structurally similar: they are described as tripartite UML packages as an incentive to build interactional components. Using Roles allows the developers to adapt these components to applicative concerns. Figure 8 presents the Interactional Object "Marker", which corresponds to the concept detailed in the above sections. It is thus possible to define the Marker's shape, colour, position, orientation or current state (locked or unlocked)...

Additionally, Interactional Objects can be organized similarly to Business Objects, through the "use" relation. As a rule, these relations can only bind Symphony Objects from the same conceptual space (i.e, interactional or business). 




Figure 9: Example of adaptation between Interactional Object and Business Object

\subsection{Adaptation modelling}

In order to illustrate the representation of business concepts into the interactional space and realize mappings between both spaces, we introduced a new "Represent" relation. Figure 9 illustrates the use of this relation, which binds the "MarkerRole" class to the "PremiseDamage" role: in our application a graphical marker is indeed responsible for representing the "Damage" concept, which is itself adapted to the "Premise" business-specific context.

The adaptation between the interactional and business spaces occurs in two steps:

Signature modelling: the adaptation realized when instantiating the Interactional Object. It is constituted by the mapping and transformation of Interactional Object attributes (and, if needed, their instanciation) to their Business Object counterpart. For instance, the "Marker" Interactional Object is adapted to the inventory of fixtures' problematic (that of a Damage Marker) by assigning it a specific colour (red) and shape (torus), through the "Represent" relation. These attributes do not have specific equivalents in the "PremiseDamage" Business Object, but correspond to the signature of this specific MarkerRole-PremiseDamage relation.

State mapping correspond to the mapping of interactive events' semantic into the business space, if it implies a modification of its corresponding Interactional Object's state. For instance, if a "DamageMarker" object (i.e., a Marker adapted to signalling damages through its static modelling specialization, see above) is set into its "locked" state, the corresponding "PremiseDamage" object must be validated (i.e., saved into the real estate agency's database). State mapping can thus be represented as statechart diagram mappings with user events triggering transitions. For the sake of conciseness, we shall not treat the specifics of these. mechanisms in this paper.

In the next section, we explore how the use of Interactional Objects and "Represent" relations eases the design "for reuse" of Interactional Components.

\section{DESIGN "FOR REUSE"}

While system modularity is central to the Symphony method, it is less trivial to organize development with reuse in mind. This is especially true for business components, which although theoretically sound, are in practice seldom developed or seldom reused as each project features a very specific approach to business.

However, by their very nature, interactional components are often reused in different applications. For instance, most of the Interactional Objects we designed (SituationalMesh, Marker, Avatar and 3DScene...) are recurrent concepts found in Augmented Reality systems.

Although we do not intend to detail design time aspects of Symphony, suffice it to say that Interactional Objects can be considered as logical abstractions from technical implementations, or Interaction Components. For instance, the Interaction Objects in the prototype we designed for the Augmented Inventory of Fixtures features an OpenGL implementation of our Interactional Components that was quite effortless to develop. In that respect, it is possible to build libraries of reusable Interactional Objects as abstractions from technical component libraries.

Moreover, aside from building single-element libraries, Interactional Objects can be provided into organized sets, for example an "Augmented Reality set" that includes commonly used Interactional Objects such as "SituationalMesh", "Avatar" and "3DScene", into which common services are described (e.g. adding an object into the scene, localizing...). Such elements could for example be reused for the MEMO system described in (Bouchet et al., 2004), for which a user wearing a HMD and using vocal commands may create virtual post-its that may be disposed anywhere (for instance, close to an interesting spot in a city) for other MEMO users to see (when visiting the aforesaid city).

Thus, Interactional Objects encourages the development of reusable components and sets of components for human-computer interaction. 


\section{CONCLUSION AND FURTHER WORK}

We have introduced in this paper a set of concepts that allows designers to take into account the design of the HCI throughout the specification and analysis phases of a design method: Symphony. While originally aimed at designing classic systems, HCI models and processes were integrated into the specification phases, during a first evolution of the method, thus permitting the design of Augmented Reality (AR) interfaces.

As a complement to these efforts, our first contribution - Interactional Objects -, allows HCI designers to describe and organize interactionspecific concepts similarly to current SE practices for the business space. Our second contribution - the "Represent" relation -, allows SE and HCI designers to draw links between the concepts that emerge in the business and interactional spaces.

Future works include finalizing the Augmented Inventory of Fixtures application, refining the method further on and applying it to a variety of projects, either in terms of domains (airport security) or type of interface (classical interaction, AR). This will also enable us to explore the reuse capacities of Interactional Objects and Interactional Components for AR as well as enlarge our component repository. Additionally, we need to explore to what extent Interactional Objects should be adapted or variable.

Finally, we plan to provide rationale concerning component choice using patterns, similarly to the approach described in (Godet-Bar et al., 2006). Refining the method shall also imply describing more formally SE and HCI model weaving.

\section{ACKNOWLEDGEMENTS}

The authors wish to thank the INPG and the IMAG federation for their financial support.

\section{REFERENCES}

Bouchet, J., Nigay, L., Ganille, T., 2004. ICARE software components for rapidly developing multimodal interfaces. In Proc. of the Sixth International Conference on Multimodal Interfaces, pages 251-258.

Chalon, R., David, B. T., 2004. Modélisation de l'interaction collaborative dans les systèmes de Réalité Mixte. In Proc. of the ACM Conference IHM'04, pages 37-44. ISBN : 1-58113-926-8. In French.
Constantine, L., Biddle, R., Noble, J., 2003. Usage-centred design and Software Engineering : Models for integration. In Proc. of the IFIP TC13 workshop on Closing the gaps: Software Engineering and HumanComputer Engineering. Borup Harning \& Jean Vanderdonckt eds.

Dubois, E., Nigay, L., Troccaz, J., 2002. Assessing continuity and compatibility in augmented reality systems. In UAIS, International Journal on Universal Access in the Information Society, Vol. 4, pages 263273. Springer-Verlag.

Dubois, E., Gauffre, G., Bach, C., Salembier, P., 2006. Participatory design meets Mixed Reality design models : Implementation based on a formal instrumentation of an informal design approach. In CADUI'06, Computer-Aided Design of User Interfaces, pages 75-88. Springer-Verlag.

Godet-Bar, G., Dupuy-Chessa, S., Nigay, L., 2006. Towards a system of patterns for the design of multimodal interfaces. In CADUI'06, Computer-Aided Design of User Interfaces, pages 27-40. SpringerVerlag.

Gulliksen, J, Göransson, B., 2005. Usability design : Integrating user-centred systems design. In The System Development Process tutorial at CHI'2005, USA.

Hassine, I., Rieu, D., Bounaas, F., Seghrouchni, O., 2002. Symphony : a conceptual model based on business components. In SMC'02, IEEE International Conference on Systems, Man, and Cybernetics, Vol. 2.

Jacobson, I., Booch, G., Rumbaugh, J., 1999. The Unified Software Development Process. Addison-Wesley.

Juras, D., Rieu, D., Dupuy-Chessa, S., 2006. Vers une méthode de développement pour les Systèmes Mixtes. In INFORSID '06, Actes du $24^{\text {ème }}$ Congrès Informatique des Organisations et Systèmes d'Information et de Décision, pages 33-48. In French.

Juras, D., Rieu, D., Dupuy-Chessa, S., Front, A., 2006. Conception collaborative pour les Systèmes Mixtes. In Revue Génie Logiciel nb.77, GL-IS, pages 31-36. In French.

Lim K. Y., Long J., 1994. The MUSE method for usability engineering, Cambridge University Press.

Paternò, F., 2003. The handbook of task analysis for human-computer interaction. Chapter ConcurTaskTrees: An Engineered Notation for Task Models, pages 483-503. Lawrence Erlbaum Associates.

Sousa, K. S., Furtado, E., 2003. An approach to integrate $\mathrm{HCI}$ and $\mathrm{SE}$ in requirements engineering. In Proc. of the IFIP TC13 workshop on Closing the gaps: Software Engineering and Human-Computer Engineering. Borup Harning \& Jean Vanderdonckt eds.

Tarby J.C., Barthet M.F., Analyse et modélisation des tâches dans la conception des systèmes d'information : la méthode Diane+. In : Analyse et conception de l'IHM, pp 117-144, Hermès, 2001. In French. 